\title{
Pesquisa clínica para o Programa Nacional de Imunizações
}

\author{
Clinical research for the Brazilian National \\ Immunization Program
}

\section{Investigación clínica para el Programa Nacional de Inmunizaciones}

\author{
Maria de Lourdes de Sousa Maia 1 \\ Patrícia Mouta Nunes de Oliveira 1 \\ Ricardo Cristiano Brum 1 \\ Letícia Kegele Lignani 1 \\ Jaqueline Toledo de Oliveira Figueira 1
}

doi: 10.1590/0102-311X00182719

\section{Introdução}

No Brasil, o Programa Nacional de Imunizações (PNI) foi criado em 1973 com o objetivo de coordenar as ações de imunizações desenvolvidas no país 1 .

O Brasil é um país de dimensões continentais, com uma diversidade geográfica bem caracterizada e fronteiras secas com muitos países da América do Sul, e isto o torna mais vulnerável às epidemias, que podem chegar ao país e se espalhar rapidamente. É portanto de suma importância termos um PNI forte nos estados e municípios brasileiros, e que consiga atingir e sustentar coberturas vacinais altas e homogêneas 1 .

Sabe-se que, com exceção da água potável, nenhuma outra medida teve tanto impacto na redução da morbimortalidade da população quanto as vacinas ${ }^{2}$. Isso pode ser comprovado pela redução das taxas de mortalidade infantil e de incidência de doenças imunopreveníveis em todo o mundo e no Brasil, após o advento da vacinação. A Figura 1 compara as incidências de algumas doenças imunopreveníveis em seu período anterior à introdução da vacinação com o período posterior. Pode-se verificar que todas as doenças mostraram queda significativa em sua incidência, comprovando o benefício da vacinação.

O PNI se destacou no cenário da América Latina comparando-se a países desenvolvidos devido ao esforço em implantar esquemas de vacinação cada vez mais completos, e que atendam às recomendações de sociedades de classe e, principalmente, ao perfil epidemiológico do país, buscando altas e homogêneas coberturas vacinais 3 .

Destaca-se a priorização que é dada a esse Programa pelo governo, demonstrada pelo seu crescente orçamento, que em 2019 ultrapassou 5 bilhões de Reais 4 . E, por ser uma ação prioritária de governo, não é passível de descontinuidade ou de cortes em seu orçamento, fato importante pois garante a continuidade do PNI, independentemente da situação política do país.

Um dos desafios desse Programa foi manter a sua sustentabilidade no que se refere ao fornecimento de imunobiológicos. Em 1985, foi criado o Programa de Autossuficiência Nacional em Imunobiológicos (PASNI), com o objetivo de ampliar a produção nacional, bem como a diversidade de vacinas produzidas no país. Estabeleceu-se um Sistema Nacional de Controle de Qualidade dos Imunobiológicos e uma parceria bem-sucedida entre o PNI e os laboratórios públicos produtores de vacinas, que perdura até hoje 1 .
1 Instituto de Tecnologia em Imunobiológicos, Fundação Oswaldo Cruz, Rio de Janeiro, Brasil.

\section{Correspondência}

M. L. S. Maia Instituto de Tecnologia em Imunobiológicos, Fundação Oswaldo Cruz.

Av. Brasil 4365, Rio de Janeiro, RJ 24040-900, Brasil.

lourdes.maia@bio.fiocruz.br 
Figura 1

Comparação entre a incidência (por 100.000 habitantes) de algumas doenças antes e após a introdução da vacinação no Brasil.

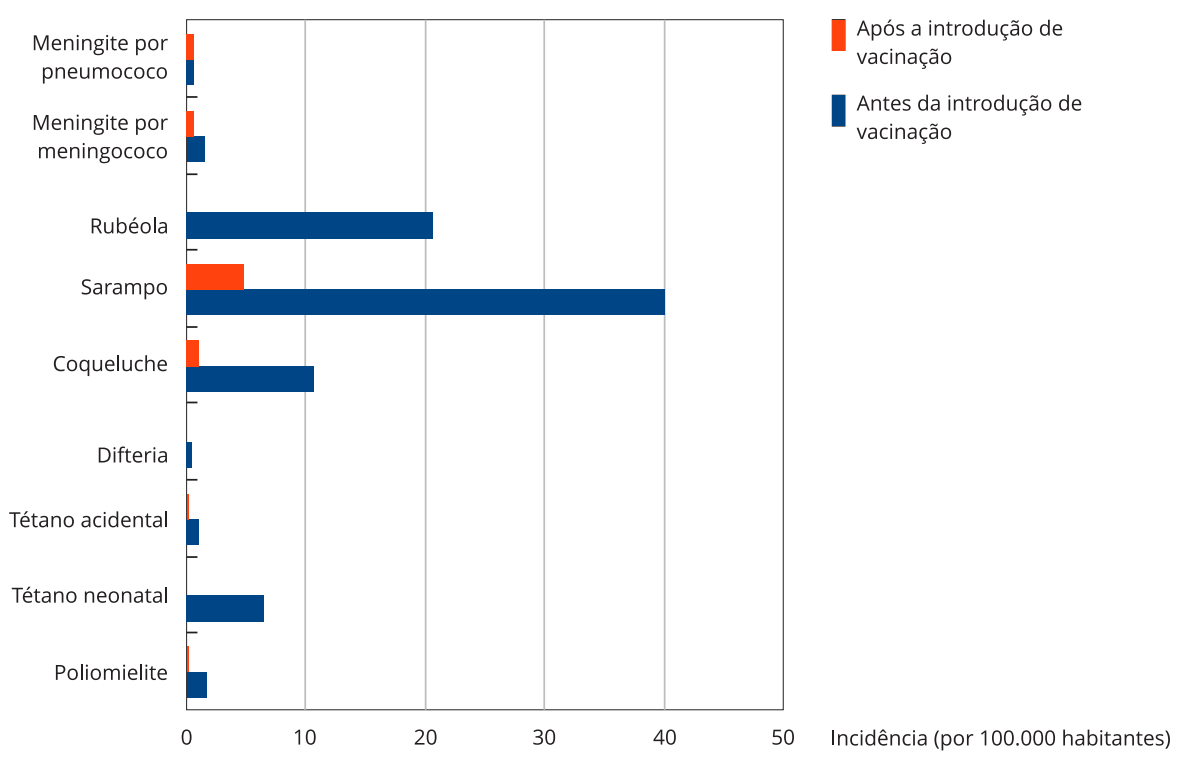

Fonte: Departamento de Imunização e Doenças Transmissíveis, Secretaria de Vigilância em Saúde, Ministério da Saúde.

Caminhando para os seus 47 anos, novos e diferentes desafios são impostos ao PNI. Um Programa que antes focava na população infantil, cresceu em suas determinações de atuar em diversos grupos etários, hoje atendendo desde recém-nascidos até idosos. É evidente que a autonomia em relação à vacinação desta nova população requer do PNI uma superação em suas estratégias, que passam por planos de comunicação, principalmente pelo momento atual das redes sociais 3 .

Esses desafios foram incrementados pelo aumento do público-alvo do Programa, com novos grupos etários; desaparecimento de doenças antes comuns, assim como a globalização trazendo novas enfermidades, levando ao aumento da diversidade e quantidade de vacinas hoje ofertadas pelo PNI. Este quadro aumentou a complexidade do calendário de vacinação e sua consequente apropriação pelos profissionais de saúde, e notadamente de sala de vacina 3 .

Aliadas a esse quadro temos as dificuldades de acesso à determinadas comunidades, seja pela questão de segurança ou crença religiosa, somando-se ao poder das mídias sociais como fonte de informação médica, espalhando muitas fake news, sendo, sem dúvida, o mais recente desafio do PNI.

Indubitavelmente, para enfrentar tão diferentes desafios o PNI precisará lançar estratégias bem diferenciadas, bem como de diversas e diferentes parcerias.

Nesse contexto, insere-se a necessidade de dar respostas de cunho cientifico que atendam à demanda de expansão de novos imunobiológicos, sejam em vacinas combinadas ou novas vacinas. Além de fornecer dados que possam embasar respostas à comunidade cientifica, usuários, grupos antivacinas e/ou combater fake news.

Demonstrar a eficácia e segurança de novas vacinas ou suas combinações pode funcionar como um elemento facilitador para melhorar a adesão pelos usuários e classe médica/científica, proporcionando confiança e consequentemente uma melhor participação nas indicações ou acompanhamento. Ademais, os responsáveis poderão se sentir mais confortáveis com as vacinas combinadas (vários antígenos aplicados em uma mesma injeção), as quais diminuem o número de injeções e retornos à unidade de saúde com consequente diminuição de custos. 
Nesse cenário, a pesquisa clínica surge como uma ferramenta fundamental para a geração de conhecimento científico. A realização de pesquisa clínica no âmbito do Sistema Único de Saúde (SUS) tem sido efetiva, não só pela população-alvo que se deseja encontrar, mas pela participação dos diferentes profissionais que se veem envolvidos e discutindo cientificamente no seu dia a dia. Com efeito, essa parceria com o SUS é uma estratégia que promove a ampliação do conhecimento e capacitação dos profissionais da assistência, além de gerar benefícios para os laboratórios públicos e fortalecer as políticas de saúde no país. Entretanto, os desafios, frente às condições das unidades de saúde, sejam pela inadequada área física ou pelo desestímulo em que os profissionais se encontram, têm sido obstáculos com os quais os profissionais de pesquisa se deparam nestas unidades; nos últimos tempos, a falta de segurança para os profissionais e usuários, os conflitos armados dentro das comunidades, que por inúmeras vezes atrasam o cronograma proposto, já que a segurança do participante e das equipes se impõe a quaisquer outros procedimentos.

Além dessas dificuldades, há ainda a questão dos tempos éticos regulatórios, que muitas vezes não se alinha com a urgência de resposta que a saúde pública necessita, mesmo utilizando todos os mecanismos legais e viáveis para acelerar esta etapa.

Sabe-se também dos custos para a realização de trabalhos de campo, material para as análises biológicas, entre outras necessidades. Para atender a essa situação, conta-se com recursos dos patrocinadores (p.ex.: Instituto de Tecnologia em Imunobiológicos, Fundação Oswaldo Cruz - Bio-Manguinhos/Fiocruz), de diversos setores do governo (Departamento de Ciência e Tecnologia, Ministério da Saúde - Decit/MS e PNI), além de agência de saúde como a Organização Mundial da Saúde (OMS) e outros. No entanto, qualquer atraso no cronograma leva ao desequilíbrio, já frágil, entre custo e financiamento.

Este artigo tem como objetivo analisar o desenvolvimento de pesquisa clínica no âmbito dos serviços públicos de saúde, a fim de prover os elementos para as decisões cientificamente fundamentadas do PNI.

\section{A experiência de Bio-Manguinhos}

Atualmente, existem sete laboratórios públicos produtores de imunobiológicos no Brasil, entre os quais: Bio-Manguinhos, o Instituto Butantan, a Fundação Ataulpho de Paiva, o Instituto de Tecnologia do Paraná (Tecpar), a Fundação Ezequiel Dias, o Instituto Vital Brazil e o Centro de Produção e Pesquisa de Imunobiológicos do Paraná 5.

A produção desses laboratórios visa a atender prioritariamente a demanda do SUS, e caso haja produção excedente a mesma pode ser fornecida a instituições, como a OMS, a Organização Pan-Americana da Saúde (OPAS) e o Fundo das Nações Unidas para a Infância (UNICEF). Para isso, as vacinas devem passar pelo processo de pré-qualificação, exigido pela OMS 6. Por intermédio do GAVI-Vaccine Alliance, parceria de entes públicos e privados que une os governos de países em desenvolvimento e de países doadores, a OMS, UNICEF, o Banco Mundial, a indústria de vacinas em países industriais e em desenvolvimento, a sociedade civil, a Fundação Bill \& Melinda Gates e outros benfeitores privados, as vacinas são distribuídas para mais de 70 países, com o objetivo de melhorar o acesso à vacinação, aumentando as coberturas vacinais das crianças que vivem nos países mais pobres do mundo 7 .

Entre os líderes em tecnologia está a Fiocruz, vinculada ao Ministério da Saúde. A entidade é responsável pelo desenvolvimento de pesquisas e fabricação de grande parte das vacinas utilizadas no PNI, do SUS 8 . A unidade da Fiocruz responsável pelo desenvolvimento, fabricação e estudos clínicos com vacinas é o Bio-Manguinhos. Conjuntamente, outros imunobiológicos e fármacos produzidos por Bio-Manguinhos, mas que escapam do escopo deste artigo, são também objeto dos estudos clínicos conduzidos pelo Instituto.

Em Bio-Manguinhos existe uma Assessoria Clínica (ASCLIN) ligada diretamente à diretoria, e que é a responsável pela condução dos ensaios clínicos com as vacinas e demais produtos fabricados pela instituição, incluindo desde estudos de fase I até a fase IV (Farmacovigilância), sempre orientados para responder às demandas provenientes de ações do SUS, e envolvendo múltiplas unidades técnicas da Fiocruz no desenvolvimento desses projetos de pesquisa. Essa área, criada em 2004, já executou mais 
de 19 estudos com vacinas, prestando um serviço estratégico para o PNI, ao desenvolver pesquisas alinhadas com as demandas e necessidades do Programa, permitindo que a população tenha acesso a imunobiológicos cada vez mais seguros e eficazes.

Dentre os estudos realizados por Bio-Manguinhos, alguns deles embasaram o PNI na tomada de importantes decisões, como por exemplo, a alteração do calendário de vacinação, com a recomendação de não aplicação simultânea das vacinas de febre amarela e tríplice viral devido à interação (com menor eficácia) entre as mesmas quando aplicadas ao mesmo tempo ou com menos de 30 dias de intervalo em menores de 2 anos 9 . Outro exemplo foi a recomendação de uso de dose fracionada da vacina de febre amarela para conter surtos/epidemias, embasada em um estudo de dose-resposta realizado por Bio-Manguinhos 10 .

Os estudos de duração da imunidade com a vacina de febre amarela, mostrando que, principalmente em crianças uma única dose não é suficiente para a proteção duradoura, subsidiaram a decisão do PNI de não seguir, inicialmente, a recomendação da OMS para a dose única da vacina e manter um reforço aos 4 anos para crianças e uma dose de reforço (10 anos após a primeira) para adultos. Essa recomendação permaneceu até a situação de surto que o país enfrentou em 2017, que obrigou o PNI a seguir, mesmo que temporariamente, a recomendação da OMS de dose única da vacina. Essa recomendação do reforço aos 4 anos para crianças será retomada em 2020, conforme Ofício-Circular no 130/2019, da Secretaria de Vigilância em Saúde do Ministério da Saúde (SVS/MS) 11.

Atualmente, Bio-Manguinhos está conduzindo dois estudos com a vacina de febre amarela, que servirão para responder às questões do PNI, sendo: um estudo de coorte em área indene e com baixo índice de processo migratório, para avaliar a duração da imunidade da vacina de febre amarela por 10 anos, e outro com a nova etapa do estudo de dose-resposta que avaliará os participantes após 10 anos da aplicação da dose reduzida da vacina. Esse último servirá para embasar a decisão do PNI de quando (e se) revacinará a população que recebeu a vacina em dose fracionada nas campanhas de 2018. Atualmente, os dados mostram proteção de até 8 anos após a aplicação da dose fracionada da vacina de febre amarela 12 .

Consequentemente, ao longo dos anos uma experiência em conduzir ensaios clínicos controlados e estudos de Farmacovigilância para o PNI foi sendo construída, e por se tratar de um cenário diferente do habitual da pesquisa clínica, várias lições foram aprendidas, o que nos permite hoje ter um maior domínio do processo de condução de pesquisa clínica para o SUS e poder descrevê-lo com alguma propriedade. Entretanto, não podemos deixar de autenticar que a aprendizagem e a melhoria são contínuas e nunca se esgotam.

\section{Como fazer pesquisa clínica em uma unidade de saúde do SUS}

Conceitualmente, um ensaio clínico tem como objetivo gerar evidências sobre a eficácia e segurança de medicamentos, e são regidos por princípios éticos baseados na Declaração de Helsinki, que devem ser a base para a aprovação e condução dos ensaios clínicos 13 .

A conduta ética é guia-mestra de todos os projetos e é assegurada pela aprovação prévia dos protocolos de pesquisa pelos Comitês de Ética em Pesquisas (CEPs) das instituições onde são realizadas. Além disso, a pesquisa clínica segue os princípios das Boas Práticas Clínicas, que são um conjunto de regulamentações, diretrizes e padrões de qualidade ética e científica, por meio dos quais os estudos envolvendo seres humanos devem ser conduzidos, sempre garantindo os direitos e o bem-estar dos participantes de pesquisa, assim como a credibilidade dos dados 14.

A RDC no 9/2015 da Agência Nacional de Vigilância Sanitária (Anvisa), que define os procedimentos e requisitos para a realização de ensaios clínicos com medicamentos no Brasil, define Centro de Ensaios Clínicos como uma organização pública ou privada, legitimamente constituída, devidamente registrada no Cadastro Nacional de Estabelecimentos de Saúde (CNES), na qual são realizados ensaios clínicos 15 .

Também, conforme o Documento das Américas e a mesma $R D C$ no 9/2015, os centros de pesquisa devem contar com instalações adequadas para a condução do protocolo clínico em relação à estrutura física, equipamentos, instrumentos e recursos humanos, e serem também adequados à população do ensaio clínico, como por exemplo, idosos, crianças, pessoas com necessidades especiais, entre outros. 
Esses locais podem ou não ser vinculados a uma universidade ou instituição de ensino e pesquisa. No caso das pesquisas envolvendo as vacinas do PNI, o ambiente ideal para a execução são as salas de vacina, que estão localizadas majoritariamente em unidades básicas de saúde 13,15.

$\mathrm{Na}$ execução de um ensaio clínico deve-se levar em conta dois cenários, um externo e um outro interno, interdependentes, e que falhas de avaliação em qualquer um deles poderão comprometer o sucesso almejado. A Figura 2 ilustra as principais etapas desse processo.

No âmbito de pesquisa clínica com vacinas para o SUS, a etapa inicial é a avaliação quanto à sua relevância para o SUS. Analisa-se a unidade de pesquisa parceira a fim de definir qual seria o melhor desenho e o melhor local (estado/município) para a realização da mesma, levando-se em conta fatores epidemiológicos, população na faixa etária desejada, logística, entre outros aspectos.

Torna-se então necessária a harmonização junto aos órgãos gestores, como Secretarias de Saúde, que podem ser a figura proponente da pesquisa, com participação de parte de sua organização, como coordenadores de áreas programáticas, programa de imunizações local, atenção primária de saúde, a Estratégia Saúde da Família (ESF) e seus agentes comunitários de saúde (ACS).

Para o êxito da pesquisa, é necessária uma apropriação do projeto pelos influenciadores locais, e a pesquisa deve ser apresentada, debatida e acordada em reunião com o Conselho Municipal e/ ou Distrital de Saúde. Entretanto, só isso não garantirá a ausência de ruídos de comunicação e nem a adesão ao projeto. Portanto, uma permanente articulação, com idas e vindas junto a esse segmento da sociedade, buscando uma construção permanente de novos saberes, é essencial para o sucesso do projeto. Planejar e executar uma pesquisa com a participação da sociedade civil organizada, alinhada com os gestores locais e equipes assistenciais, é o que vem garantindo o êxito.

\section{Figura 2}

Processo para execução de um estudo clínico no Sistema Único de Saúde (SUS).

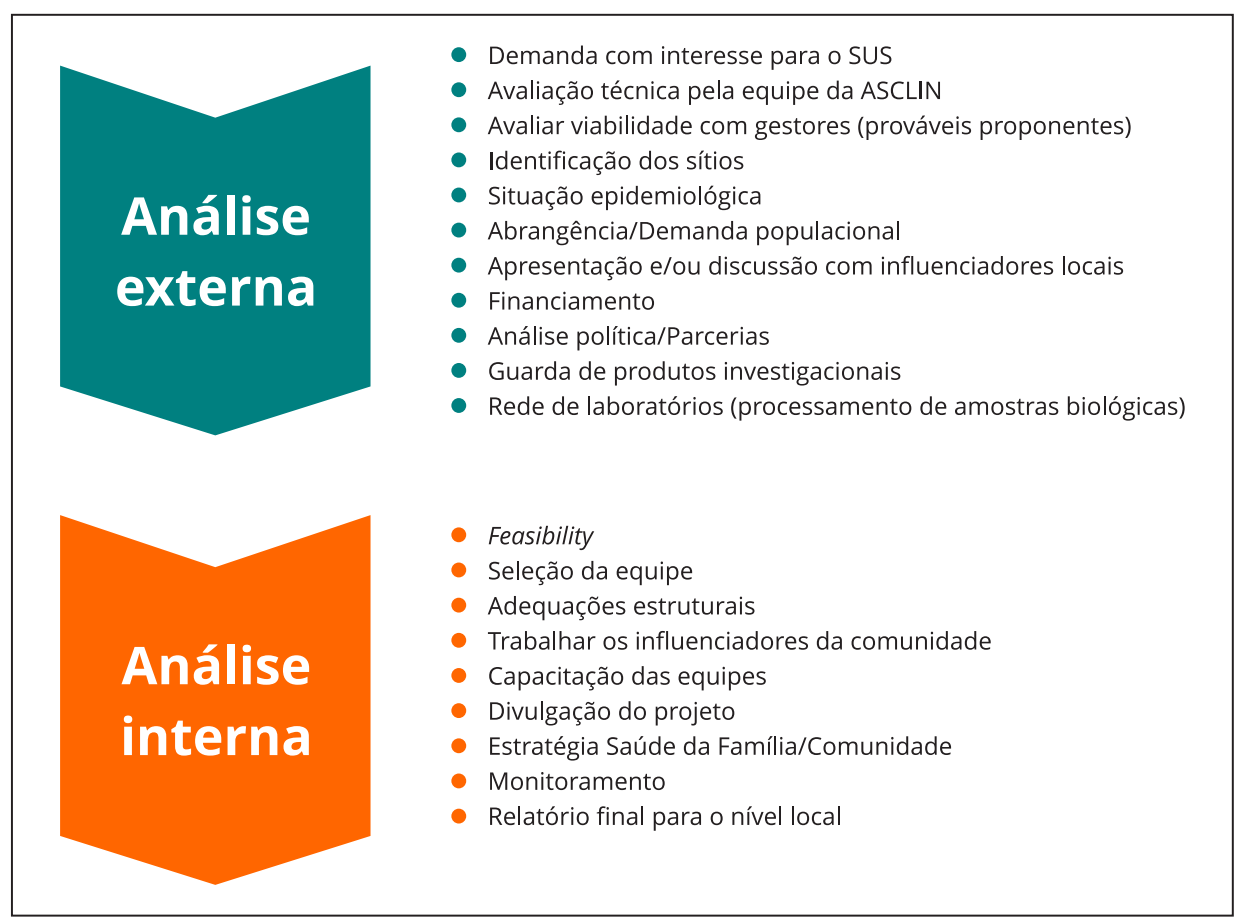

ASCLIN: Assessoria Clínica, Instituto de Tecnologia em Imunobiológicos, Fundação Oswaldo Cruz. 
Um exemplo de projeto conduzido nesses moldes é o estudo de duração da imunidade com a vacina de febre amarela - coorte de 10 anos - em área indene para esta febre, que está sendo conduzido por Bio-Manguinhos em três municípios da Paraíba. O estudo começou em julho de 2016 no Município de Alhandra e posteriormente nos municípios de Conde e Caaporã, com um total de 4.761 participantes captados. Na etapa de planejamento foi necessário identificar, mobilizar e fechar parcerias com diversas instituições como Secretaria Estadual de Saúde, prefeituras e secretarias municipais, Universidade Federal da Paraíba, Sociedade Paraibana de Pediatria e de Imunizações, Conselho Regional de Medicina, entre outros. A participação de movimentos sociais e a ESF estão sendo essenciais para a condução do estudo, com o apoio e mobilização dos ACS dos municípios. Foram 111 ACS em 18 localidades dos 3 municípios na captação dos 2.005 adultos e 2.756 crianças. Como o trabalho tem duração de 10 anos, a parceria e apoio desses profissionais continua sendo essencial para a continuidade do estudo (dados da Assessoria Clínica de Bio-Manguinhos não publicados).

O reconhecimento do cenário de atuação deve fazer parte do planejamento, desde suas áreas de abrangência e influência até os influenciadores locais. Ter agentes de mobilização com estabelecida credibilidade, relação de confiança dentro da comunidade e inserir o participante da pesquisa nas discussões do projeto, sempre que possível, é mister para que a pesquisa não seja vista como inclusão de "cobaias" e sim como uma participação da sociedade na sustentabilidade do SUS.

Deve-se garantir o orçamento necessário para a realização do estudo, que deverá constar na documentação enviada ao Comitê de Ética. A garantia de continuidade de um projeto está atrelada às Boas Práticas Clínicas, que enfatiza os princípios éticos da não maleficência e de beneficência para o participante de pesquisa 13 .

Outro ponto importante é a divulgação interna do projeto no centro de pesquisa. É preciso envolver toda a equipe do centro no estudo, para que a adesão seja efetiva. Muitas vezes é difícil reunir toda a força de trabalho da unidade de saúde para uma apresentação. Nesses casos, deve-se reunir separadamente com as áreas estratégicas, como por exemplo, recepção, pediatras (em caso de estudos com crianças), enfermagem, entre outras. Outra estratégia para garantir a divulgação interna é utilizar postagens para grupos de troca de mensagens (p.ex.: WhatsApp) de profissionais de saúde da unidade, notícias no site da unidade, além, é claro, dos já conhecidos cartazes. Lembrando que todo material de divulgação deve ser submetido e aprovado pelo Comitê de Ética 14.

Em relação à infraestrutura, uma unidade básica de saúde, na maioria das vezes, não dispõe de todo o aparato exigido para a condução de um estudo clínico. Por isso, é realizado um diagnóstico situacional das unidades (feasibilty) e, se aplicável, melhorias devem ser feitas na infraestrutura para adequá-las às Boas Práticas Clínicas. Um exemplo de adequação muitas vezes necessária é a instalação de Internet, permitindo acesso aos programas de e-CRF (Electronic Case Report Form), Geclin (sistema da Assessoria Clínica que permite o gerenciamento remoto dos estudos clínicos), a adequação de salas para atendimento de participantes, coleta e guarda de material, entre outros. Destacam-se aqui as dificuldades encontradas em alguns locais para garantir uma Internet de qualidade. Isso ocorre até em grandes capitais, como Rio de Janeiro. Para minimizar esse problema, Bio-Manguinhos desenvolveu, em parceria com o Serviço Federal de Processamento de Dados (SERPRO; http://www.serpro.gov.br), um e-CRF totalmente em português, customizável e agora já na versão off-line.

Uma outra área estratégica é a Rede de Frio, que deve guardar os produtos sob investigação e de referência, no caso de uma pesquisa de intervenção. Nesse aspecto, não basta uma câmara frigorífica exclusiva e validada para o estudo clínico. Necessita-se de rígido controle de temperatura, com rastreabilidade e possibilidade de detectar imediatamente falha, uma vez que muitas unidades não dispõem de geradores de energia. A detecção imediata de excursões de temperatura, por exemplo, permite o acionamento do plano de contingência para estas situações, e constitui um dos documentos do estudo, servindo para mitigar possíveis falhas 16 .

A seleção dos recursos humanos é mais uma etapa fundamental nesse processo. O ideal é que sejam priorizados colaboradores que já atuem na unidade de saúde e tenham carga horária disponível para o projeto, ou que já tenham atuado na unidade anteriormente (p.ex.: aposentados). Nossa experiência mostra que trabalhar com uma equipe familiarizada com o local e com sua população faz a diferença na execução de uma pesquisa clínica.

Um ponto importante a ressaltar é o ganho adicional para a unidade onde é desenvolvida a pesquisa clínica, o que fica evidente seja pela experiência de nossa equipe, seja pelos testemunhos de 
profissionais que já atuaram nos projetos. Notadamente, a execução das atividades de pesquisa clínica leva a impactos positivos para a equipe local, com melhorias no processo de trabalho, além de novas perspectivas para esses profissionais sobre suas atuações na sociedade. Na experiência dos autores, vários profissionais que trabalharam em ensaios clínicos começaram ou retomaram sua vida acadêmica após a experiência com pesquisa clínica.

\section{Dificuldades e desafios}

Como pôde ser observado, vários desafios estão postos para o PNI e para todos que dão suporte ao Ministério da Saúde e ao SUS. Estratégias cada vez mais eficientes e rápidas precisam ser desenvolvidas para permitir que as respostas à população e à comunidade científica sejam embasadas em dados robustos e confiáveis.

Por isso, a pesquisa clínica vem se tornando cada vez mais uma ferramenta necessária e eficaz para atender às demandas do PNI. Ter a possibilidade e o conhecimento para executar estudos localmente, que respondam às questões estratégicas para o Programa é um ganho extraordinário, pois gera informação científica específica para nossos produtos, nossa população e nossa realidade.

Entretanto, realizar pesquisas clínicas seguindo as Boas Práticas Clínicas em unidades básicas de saúde do SUS representou um enorme desafio. Ao longo dos anos, as pesquisas clínicas realizadas por Bio-Manguinhos puderam chegar a 7 estados do Nordeste, 2 do Norte, 2 do Centro-oeste, 3 do Sudeste e 2 do Sul, mais o Distrito Federal, cobrindo 58\% dos estados brasileiros, retratando assim, mais uma importante contribuição à saúde pública, uma vez que insere outras regiões fora do eixo Sul e Sudeste no cenário da pesquisa clínica, mostrando que é possível fazer um trabalho de qualidade e gerar melhorias tanto nas unidades básicas de saúde (UBS) onde os estudos aconteceram, como para os profissionais que atuam nos mesmos.

Notamos que um dos grandes fatores que dificulta a realização de pesquisas em regiões mais distantes dos grandes centros urbanos são as questões logísticas e orçamentárias, já que os custos de deslocamento das equipes, transporte de amostras e produto investigacional aumentam o orçamento do projeto. Entretanto, esforços devem ser feitos para que as pesquisas clínicas possam chegar às diferentes regiões, o que muitas vezes é necessário dentro da epidemiologia desejada.

Outro ponto sensível é a captação de recursos humanos para essas regiões devido à falta de capacitação de profissionais em pesquisa clínica fora dos grandes centros. No entanto, essa deficiência tem sido superada com o treinamento e acompanhamento de profissionais locais, qualificando equipes para projetos futuros.

Alinhar os tempos ético-regulatórios com as pesquisas de interesse para a saúde pública e conseguir fontes de financiamento são fatores que sabemos ser necessários para um trabalho mais efetivo e com menor tempo de resposta.

O espaço físico também representa um desafio, visto que competimos com o atendimento de rotina do centro de saúde, pois os fluxos da pesquisa quase sempre não correspondem à realidade da rotina. No entanto, com a parceria das unidades de saúde estamos conseguindo vencer esses obstáculos por meio de revisão de fluxos de trabalho e otimização de espaços, adaptando-os dentro das Boas Práticas Clínicas. Esse processo passa pela aquisição de equipamentos e mobiliários que a cada estudo realizado precisam ser adquiridos e, na maioria das vezes, ao final do trabalho são doados à unidade onde o estudo ocorreu, gerando um custo.

Diante disso, a ASCLIN, em parceria com a Secretaria Municipal de Saúde do Rio de Janeiro, tem investido na montagem de centros de pesquisa fixos dentro de algumas unidades de saúde do SUS. São espaços projetados e destinados exclusivamente para ensaios clínicos, atendendo rigorosamente aos requisitos de Boas Práticas Clínicas. Atualmente, estão em funcionamento dois centros de pesquisa, um no Centro Municipal de Saúde Heitor Beltrão, na Tijuca, e outro na Policlínica Lincoln de Freitas Filho, em Santa Cruz, ambos no Município do Rio de Janeiro. Esse último conta também com um Laboratório de Processamento de Amostras Clínicas nível de Biossegurança NB2 17. 


\section{Conclusão}

A pesquisa clínica em unidades do SUS é uma ferramenta estratégica para o PNI, gerando conhecimento científico sobre as vacinas oferecidas e, com isto, mantendo a confiança da população e dos profissionais de saúde no Programa.

Além disso, a execução de um estudo clínico em uma unidade básica de saúde traz oportunidades de melhoria para os processos de trabalho, já que todas as pesquisas devem ser desenvolvidas de acordo com as Boas Práticas Clínicas. Contribui também para a formação de profissionais capacitados em pesquisa clínica, podendo aumentar a contribuição do Brasil em projetos de pesquisa.

A oportunidade de se fazer pesquisa clínica para o PNI surge como um instrumento de fortalecimento das políticas de saúde do SUS, pois além de demonstrar a eficácia e segurança de novas vacinas de interesse epidemiológico, promove o aumento e disponibilidade de produtos que atendam às demandas do Ministério da Saúde.

As pesquisas realizadas fornecem dados seguros na condução de novas orientações e manejos em saúde, como os estudos de duração da imunidade com a vacina de febre amarela, mostrando que, principalmente em crianças, uma única dose não é suficiente para a proteção duradoura.

Como desafios para a realização de pesquisas em algumas regiões do país, precisamos melhorar as condições logísticas e orçamentárias, que incluem o controle rigoroso da rede de frio, capacitações etc. Em linhas gerais, a pesquisa clínica para o SUS vem ganhando cada vez mais robustez e fornecendo contribuições científicas valiosas para o Ministério da Saúde, além de fortalecer as políticas junto aos laboratórios públicos, incluindo Bio-Manguinhos.

Sabe-se que ainda existe um longo caminho pela frente no que tange ao estabelecimento de parcerias, que devem envolver as associações de moradores, conselhos de saúde, atores de mobilização, sociedades de classe, e o grande desafio: o participante de pesquisa. Para isso, novas estratégias e políticas precisarão ser estabelecidas e reforçadas.

Dessa forma, a pesquisa clínica no âmbito do PNI, alinhada aos interesses e demandas do Ministério da Saúde, consiste num importante passo para o avanço das pesquisas no Brasil, motivando interesses e promovendo o desenvolvimento do setor de saúde e a melhoria da qualidade de saúde da população brasileira.

\section{Colaboradores}

Todos os autores contribuíram na concepção, elaboração, revisão e aprovação da versão final do artigo.

\section{Informações adicionais}

ORCID: Maria de Lourdes de Sousa Maia (00000003-4223-9151); Patrícia Mouta Nunes de Oliveira (0000-0001-6867-398X); Ricardo Cristiano Brum (0000-0001-9732-8042); Letícia Kegele Lignani (0000-0001-9066-5230); Jaqueline Toledo de Oliveira Figueira (0000-0002-7960-7344).

\section{Conflito de interesses}

Todos os autores trabalham em Bio-Manguinhos, produtor de vacinas para o PNI.

\section{Agradecimentos}

Agradecemos ao Decit/MS e ao PNI. 


\section{Referências}

1. Departamento de Vigilância das Doenças Transmissíveis, Secretaria de Vigilância em Saúde, Ministério da Saúde. Programa Nacional de Imunizações (PNI): 40 anos. Brasília: Ministério da Saúde; 2013.

2. Plotkin SL, Plotkin AS, Orenstein W, Offit PA, Orenstein WA, Edwards KM. Plotkin's vaccines. 17th Ed. Philadelphia: Elsevier; 2018.

3. Associação da Indústria Farmacêutica de Pesquisa. Como as vacinas mudaram um país. https://www.interfarma.org.br/public/files/ biblioteca/como-as-vacinas-mudaram-umpais-interfarma.pdf (acessado em 27/Dez/ 2019).

4. Ministério da Economia, Planejamento, Desenvolvimento e Gestão. Orçamento da União. Exercício financeiro de 2019. http://www. planejamento.gov.br/assuntos/orcamento-1/ orcamentos-anuais/2019/loa-2019/volume-i. pdf/view (acessado em 11/Jul/2019).

5. Soares PF. Compreendendo se e como os condicionantes dos laboratórios públicos produtores de imunobiológicos no Brasil interferem na atuação do gestor público [Tese de Doutorado]. Rio de Janeiro: Instituto Alberto Luiz Coimbra de Pós-Graduação e Pesquisa de Engenharia, Universidade Federal do Rio de Janeiro; 2012.

6. World Health Organization. Essential medicines and health products: prequalification of medicines. https://extranet.who.int/prequal/ (acessado em 11/Jul/2019).

7. GAVI-Vaccine Alliance. Gavi's mission. https://www.gavi.org/about/mission/ (acessado em 11/Jul/2019).

8. Brasil é referência mundial na fabricação vacinas. http://www.brasil.gov.br/noticias/edu cacao-e-ciencia/2010/12/brasil-e-referenciamundial-na-fabricacao-vacinas (acessado em 14/Jul/2019).

9. Silva JRN, Camacho LAB, Siqueira MM, Freire MS, Castro YP, Maia MLS, et al. Mutual interference on the immune response to yellow fever vaccine and a combined vaccine against measles, mumps and rubella. Vaccine 2011; 29:6327-34.
10. Martins RM, Maia MLS, Farias RHG, Camacho LAB, Freire MS, Galler R, et al. 17DD yellow fever vaccine: a double blind, randomized clinical trial of immunogenicity and safety on a dose-response study. Hum Vaccin Immunother 2013; 9:879-88.

11. Secretaria de Vigilância em Saúde, Ministério da Saúde. Ofício-Circular no 130/2019/SVS/ MS. https://sbim.org.br/informes-e-notastecnicas/outras-entidades (acessado em 27/ Dez/2019).

12. Martins RM, Maia MLS, Lima SMB, Noronha TG, Xavier JR, Camacho LAB, et al. Duration of post-vaccination immunity against yellow fever in adults. Vaccine 2018; 36:4112-7.

13. Organização Pan-Americana da Saúde. Manual de boas práticas clínicas: documentos das Américas. Santo Domingo: Organização Pan-Americana da Saúde; 2005.

14. Conselho Nacional de Saúde. Resolução no 466, de 12 de dezembro de 2012. Diário Oficial da União 2013; 13 jun.

15. Agência Nacional de Vigilância Sanitária. Resolução da Diretoria Colegiada - RDC no 9, de 20 de fevereiro de 2015. Dispõe sobre o Regulamento para a realização de ensaios clínicos com medicamentos no Brasil. Diário Oficial da União 2015; 3 mar.

16. Cardoso AS, Costa CB, Barros CP, Furtado FS, Estery JLB. Gestão da rede de frio de imunibiológicos. In: Silva MN, Flauzino RF, organizadoras. Rede de frio: gestão, especificidades e atividades. Rio de Janeiro: Editora Fiocruz; 2017. p. 175-223.

17. Agência Nacional de Vigilância Sanitária. Microbiologia clínica para o controle de infecção relacionada à assistência à saúde. Módulo 1: biossegurança e manutenção de equipamentos em laboratório de microbiologia clínica. Brasília: Agência Nacional de Vigilância Sanitária; 2013.
Recebido em 20/Set/2019

Versão final reapresentada em 06/Jan/2020

Aprovado em 31/Jan/2020 